\title{
ZmWRKY104 Transcription Factor Phosphorylated by ZmMPK6 Functioning in ABA-Induced Antioxidant Defense and Enhance Drought Tolerance in Maize
}

\author{
Lili Zhao ${ }^{1}$, Jingwei Yan ${ }^{1}$, Yang Xiang ${ }^{1}$, Yue Sun ${ }^{1}$ and Aying Zhang ${ }^{1,2, *}$ \\ 1 College of Life Sciences, Nanjing Agricultural University, Nanjing 210095, China; \\ 2016216025@njau.edu.cn (L.Z.); jingweiyan@njau.edu.cn (J.Y.); 2017216005@njau.edu.cn (Y.X.); \\ sunyueacer@163.com (Y.S.) \\ 2 State Key Laboratory of Crop Genetics and Germplasm Enhancement, Nanjing Agricultural University, \\ Nanjing 210095, China \\ * Correspondence: ayzhang@njau.edu.cn; Tel.: +86-25-8439-9078
}

check for updates

Citation: Zhao, L.; Yan, J.; Xiang, Y.; Sun, Y.; Zhang, A. ZmWRKY104 Transcription Factor Phosphorylated by ZmMPK6 Functioning in ABA-Induced Antioxidant Defense and Enhance Drought Tolerance in Maize. Biology 2021, 10, 893. https://doi.org/10.3390/biology 10090893

Academic Editor: Daniel G. Peterson

Received: 2 August 2021

Accepted: 7 September 2021

Published: 10 September 2021

Publisher's Note: MDPI stays neutral with regard to jurisdictional claims in published maps and institutional affiliations.

Copyright: (c) 2021 by the authors. Licensee MDPI, Basel, Switzerland. This article is an open access article distributed under the terms and conditions of the Creative Commons Attribution (CC BY) license (https:/ / creativecommons.org/licenses/by/ $4.0 /)$.
Simple Summary: Current knowledge about the downstream substrate proteins of MAPKs is still limited. Our study screened a new WRKY Ila transcription factor as the substrate protein of ZmMPK6, and its phosphorylation at Thr-59 is critical to the role of ZmWRKY104 in ABA-induced antioxidant defense. Moreover, overexpression ZmWRKY104 in maize enhances the drought tolerance of transgenic plants. These findings define a mechanism for the function of ZmWRKY104 phosphorylated by ZmMPK6 in ABA-induced antioxidant defense and drought tolerance.

Abstract: Mitogen-activated protein kinase (MAPK) cascades are primary signaling pathways involved in various signaling pathways triggered by abiotic and biotic stresses in plants. The downstream substrate proteins of MAPKs in maize, however, are still limited. Here, we screened a WRKY IIa transcription factor (TF) in maize (Zea mays L.), ZmWRKY104, and found that it is a substrate of ZmMPK6. ZmWRKY104 physically interacts with ZmMPK6 in vitro and in vivo. Liquid chromatography-tandem mass spectrometry (LC-MS/MS) analysis results showed that threonine59 (Thr-59, T59) was the major phosphorylation site of ZmWRKY104 by ZmMPK6. Subcellular localization analysis suggested that ZmWRKY104 acts in the nucleus and that ZmMPK6 acts in the nucleus and cytoplasmic membrane in the cytosol. Functional analysis revealed that the role of ZmWRKY104 in ABA-induced antioxidant defense depends on ZmMPK6. Moreover, overexpression of ZmWRKY104 in maize can enhance drought tolerance and relieve drought-induced oxidative damage in transgenic lines. The above results help define the mechanism of the function of ZmWRKY104 phosphorylated by ZmMPK6 in ABA-induced antioxidant defense and drought tolerance in maize.

Keywords: WRKY transcription factor; mitogen-activated protein kinase (MAPK); abscisic acid (ABA); phosphorylation; antioxidant defense; drought stress; maize

\section{Introduction}

Abiotic stresses such as drought, salinity, oxidative stress, and temperature variations change the productivity of major crops and constitute the main cause of global crop losses [1,2]. Maize (Zea mays L.) is a major cereal crop species worldwide that is severely impacted by various abiotic stresses during its growth and productivity. Abiotic stresses also activate the emergence of reactive oxygen species (ROS) in plant cells. Abscisic acid (ABA) serves as a key endogenous messenger in the abiotic stress responses of plants [3], and can induce antioxidant defense-related enzymes such as ascorbate peroxidase (APX), superoxide dismutase (SOD), glutathione peroxidase (GPX) and catalase (CAT) [4]. Many signal molecules, such as calcium ion, nitric oxide (NO), and protein kinases, such as calcium-dependent protein kinase (CDPK), calcium/calmodulin-dependent protein kinase 
(CCaMK), and mitogen-activated protein kinase (MAPK), are involved in ABA signal response [5-7]. Besides, ABA can regulate plant growth, photosynthesis, and the synthesis of phenolic compounds $[8,9]$. Understanding ABA-induced antioxidant defense is essential for improving plant performance under abiotic stress.

The prototypical MAPK phosphorylation cascade is composed of a set of three evolutionarily conserved MAPKs, namely, MAPK kinase kinase (MAPKKK, MEKK), MAPK kinase (MAPKK, MEK), and MAPK, which play important roles in plant growth, development, and defense responses [10]. Activated MAPK phosphorylates and activates downstream substrate, such as transcription factors (TFs) at conserved amino acid residues (S/T-P motif). MAPK cascades not only play important roles in the regulation of many biological processes in plants but are also activated by drought, salinity, cold, wounding, ROS, and hormone stimuli [11]. According to their sequence homology and conserved phosphorylation motifs, MAPKs have been categorized into four major groups. Members of groups A and B have been extensively reported, and increasing knowledge about Group C MAPKs has emerged [12-16], but few studies have investigated the function of MAPKs belonging to group D. Group D MAPKs carry a T-D-Y phosphorylation motif. Gossypium hirsutum MPK16, which was the first group D MAPK gene found in cotton, is involved in drought sensitivity [12]. MPK18, Arabidopsis group D MAPK, involved in microtubulerelated functions [17]. ZmMPK17, a maize group D MAPK gene, is in response to ABA, hydrogen peroxide, salicylic acid, jasmonic acid, ethylene, low temperature, and osmotic stress [18]. ZmMPK6 is a member of group D of plant MAPKs and interacts with 14-3-3 proteins in vitro, but its function has not been defined [19].

As one of the largest families of transcriptional regulators in higher plants, WRKY TFs form integral components of signaling networks that modulate various plant processes $[20,21]$. The characteristic feature of the WRKY superfamily is the presence of a highly conserved WRKY domain containing the almost invariant WRKYGQK peptide at the $\mathrm{N}$-terminus and zinc-finger motifs at the $\mathrm{C}$-terminus [22]. According to the single finger motif of a small subset of WRKY proteins, WRKYs are divided into groups I, II, and III [20]. Building on the primary amino acid sequence, the WRKY group II family is further separated into IIa, Ilb, IIc, IId, and IIe [23]. It has been shown that the function of most WRKY TFs in regulating transcriptional reprogramming associated with plant abiotic responses such as salt, drought, and cold. A study showed that activated expression of AtWRKY57 could improve drought tolerance of Arabidopsis by elevation of ABA levels [24]. AtWRKY46 regulated responses to osmotic/salt stress in Arabidopsis [25]. TaWRKY1 and TaWRKY33 were involved in abnormal responses to drought and ABA [26]. AtWRKY46, AtWRKY54, and AtWRKY70, are involved in plant growth and drought response [27]. GmWRKY12 was highly induced by drought and salt treatments [28]. OsWRKY IIa subfamily members (OsWRKY62, OsWRKY28, OsWRKY71, and OsWRKY76) modulate innate immunity in rice [29]. Barley WRKY IIa transcripts HvWRKY1/2 suppress the basal defense mechanism against virulent Blumeria graminis, as demonstrated by silencing and transient overexpression experiments [30,31]. Arabidopsis WRKY18, WRKY40, and WRKY60 are involved in a complex pattern in plant responses to ABA and abiotic stresses [32]. WRKY TFs can be activated through several pathways, such as phosphorylation by mitogen-activated protein kinases (MPKs) [33,34]. Despite the functional characterization of WRKY TFs activated by MAPKs in Arabidopsis and rice [35,36], WRKY TFs in maize have not been studied extensively.

In the current study, we identified a WRKY IIa TF, ZmWRKY104, as the substrate protein of ZmMPK6 in maize, investigated the interaction between ZmWRKY104 and $Z m M P K 6$, and analyzed the roles of ZmWRKY104 in ABA-induced antioxidant defense and drought tolerance in maize.

\section{Results}

\subsection{Identification and Sequence Analysis of ZmWRKY104 and ZmMPK6}

The full-length coding DNA sequence (CDS) of ZmWRKY104 (GRMZM2G169149), which encodes a polypeptide consisting of 266 amino acid residues, was obtained from 
the Maize GDB (https:/ / maizegdb.org/, accessed on 1 May 2020). Conserved domain analysis revealed that ZmWRKY104 has a highly conserved WRKYGQK motif and C2H2 (C-X5-C-X23-H-X1-H) zinc finger motif (Figure S1A). Multiple alignment shows that WRKY was highly conserved among A. thaliana, O. sativa, and Z. mays (Figure S1B). Phylogenetic analysis showed that ZmWRKY104 belongs to the WRKY IIa subfamily with a single WRKY domain, and is highly homogeneous to OsWRKY62 (Figure S2) [37]. In a previous study, we screened the possible interacting proteins of ZmWRKY104 and identified a serine/threonine protein kinase-ZmMPK6. The full-length cDNA sequence of ZmMPK6 (NP_001105238) was obtained from GenBank (https://www.ncbi.nlm.nih.gov/genbank/, accessed on 1 May 2020), which encodes a polypeptide of 557 amino acid residues and a potential S_TKc. Multiple alignment revealed that MAPK was greatly conserved among $O$. sativa, A. thaliana, G. hirsutum, T. aestivum, and Z. mays, and a phylogenetic analysis showed that ZmMPK6 was highly homogeneous to OsMPK15 (Figure S3B). ZmMPK6 belongs to Group D of plant MAPKs due to the presence of the T-D-Y activation motif in the T-loop via an extended C-terminal region (Figure S3A) [19].

\subsection{ZmWRKY104 Is a Positive Regulator in ABA-Induced Antioxidant Defense}

We first assessed the expression patterns of ZmWRKY104 in different tissues of maize. We isolated total RNA from different tissues, including roots, stems, leaves, pollen, and pistil. The results of qRT-PCR showed that ZmWRKY104 were expressed in all tested tissues, and the expression was the highest in leaves (Figure S4). The expression of ZmWRKY104 increased at $15 \mathrm{~min}$ and reached a maximum at $90 \mathrm{~min}$, with a sevenfold change, and maintained for up to $240 \mathrm{~min}$ after treatment in maize leaves, which suggested an association of this TF with the response to ABA (Figure 1A). To investigate the part of ZmWRKY104 in ABA-induced antioxidant defense, we determined the effect of the transient expression or silencing of ZmWRKY104 in maize mesophyll protoplasts $[38,39]$ on the activities of primary enzymes of APX and SOD in antioxidant defense. Firstly, we detected the efficiency of transiently overexpressing ZmWRKY104, silencing ZmWRKY104, and $s r d x Z m W R K Y 104$ in maize protoplasts. The results showed that compared with the control, the gene expression level of ZmWRKY104 was increased by about 3.5-fold after transient transformation (Figure 1B). After transient transformation of ZmWRKY104RNAi (Figure 1C) and srdxZmWRKY104 (Figure 1B), compared with the control, the gene expression level decreased by about $50 \%$, indicating that the transient transformation of protoplasts can achieve the expected effect. As shown in Figure 1D,E, the activities of APX and SOD after transient expression of ZmWRKY104 were significantly higher than those in the control, and ABA treatment further enhanced these increased activities. On the contrary, transient silencing of ZmWRKY104 significantly reduced its activities compared with the control, and ABA treatment could not return these decreased activities to baseline levels (Figure 1F,G). WRKY TFs have functional redundancy [40]. To validate the function of ZmWRKY104, chimeric repressor silencing technology was used [7,24,25]; we fused ZmWRKY104-SRDX-mCherry to the exogenous EAR motif repression domain SRDX and transformed the product (srd $x$ ZmWRKY104) into maize protoplasts. Similarly, transient expression of $s r d x Z m W R K Y 104$ also resulted in significant decreases in the activities of APX and SOD, and ABA treatment could not return their activities to the control levels (Figure 1H,I). In summary, all the above-described results provide clear facts that $Z m W R K Y 104$ is a positive regulator in ABA-induced antioxidant defense. 


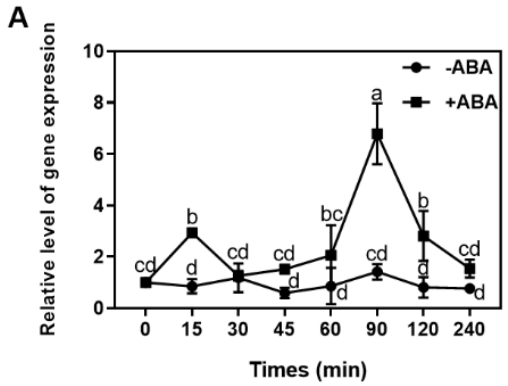

D



$E$

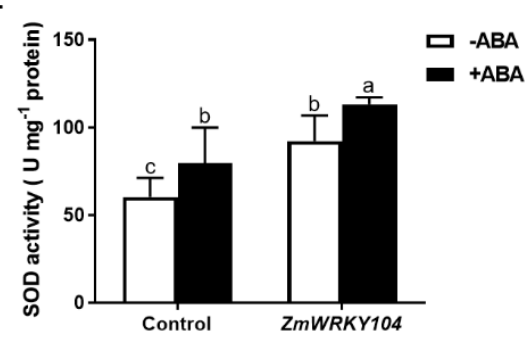

B

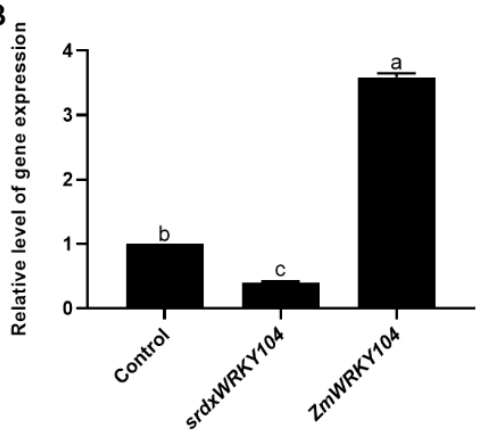

$\mathrm{F}$

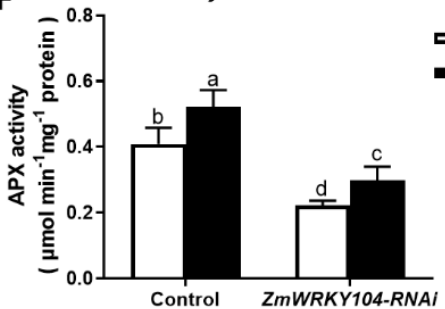

G

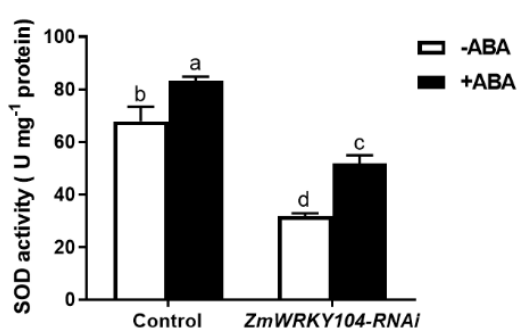

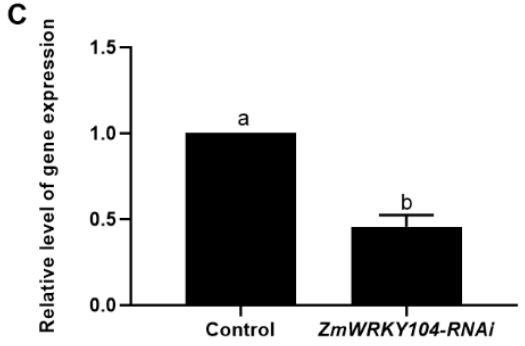

H

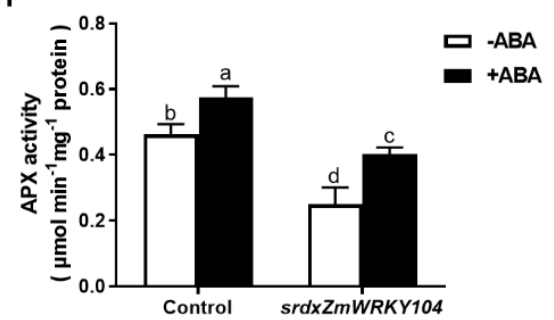

I

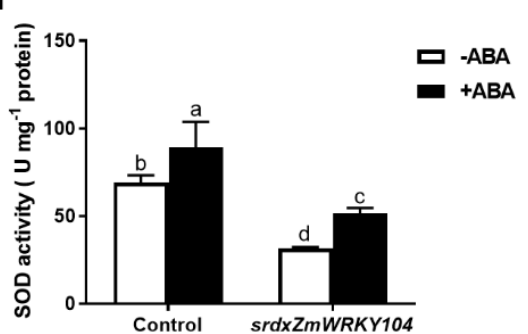

Figure 1. ZmWRKY104 is related to ABA-induced antioxidant defense. (A) Expression levels of ZmWRKY104 in maize leaves treated with ABA. The separated maize plants were treated with $100 \mu \mathrm{M}$ ABA for different times. qRT-PCR detect the expression levels of ZmWRKY104 relative to ZmActin; (B) detection of the efficiency of transiently overexpressing ZmWRKY104 and srdxZmWRKY104 in maize protoplasts; (C) detection of the efficiency of transiently silencing ZmWRKY104 in maize protoplasts. Gene expression analysis of ZmWRKY104 by RT-qPCR, with ZmActin as an internal control. Activities of APX (D) and SOD (E) of transiently expressing ZmWRKY104 in protoplasts. ZmWRKY104 (ubi: ZmWRKY104-mCherry) or Control (empty vector) were transfected into protoplasts. Activities of APX (F) and SOD (G) of transiently silencing ZmWRKY104 in protoplasts. ZmWRKY104-RNAi or Control (distilled water) were transfected into protoplasts. Activities of APX (H) and SOD (I) of transiently expressing srdxZmWRKY104 in protoplasts. srdxZmWRKY104 (ubi: ZmWRKY104SRDX-mCherry) or Control (empty vector) were transfected into protoplasts. Culture medium (-ABA) or $10 \mu \mathrm{M} A B A$ $(+\mathrm{ABA})$ were treated with the protoplasts for $10 \mathrm{~min}$. Values are the means $\pm \mathrm{SE}$ of three different experiments. According to Duncan's multiple range test, means represented by the same letter did not significantly differ at $p<0.05$.

\subsection{ZmWRKY104 and ZmMPK6 Function in ABA-Induced Antioxidant Defense}

Our previous research reported that MAPK was related to ABA-induced antioxidant defense [6]. Thus, we wondered whether ZmWRKY104 and ZmMPK6 function together in ABA-induced antioxidant defense. As shown in Figure 2, the activities of APX and SOD after transient expression of ZmWRKY104 or ZmMPK6 alone were significantly increased, and these increased activities were further enhanced by ABA treatment. Compared with ZmWRKY104 or ZmMPK6 alone, co-expression of ZmWRKY104 and ZmMPK6 further increased the activities. The activities of APX and SOD were also further enhanced by treatment with ABA. These results suggest that co-expression of ZmWRKY104 and ZmMPK6 improves ABA-induced antioxidant defense in maize.

Moreover, we investigated the subcellular localizations of ZmWRKY104 and ZmMPK6. ZmWRKY104 and ZmMPK6 fused to yellow fluorescent protein (YFP) were transiently expressed by Agrobacterium infiltration in tobacco leaves. Subcellular localization results showed that ZmWRKY104 was specifically located in the nucleus of tobacco mesophyll 
cells, and ZmMPK6 was specifically located in the cytoplasmic membrane and nucleus of tobacco mesophyll cells (Figure 3).
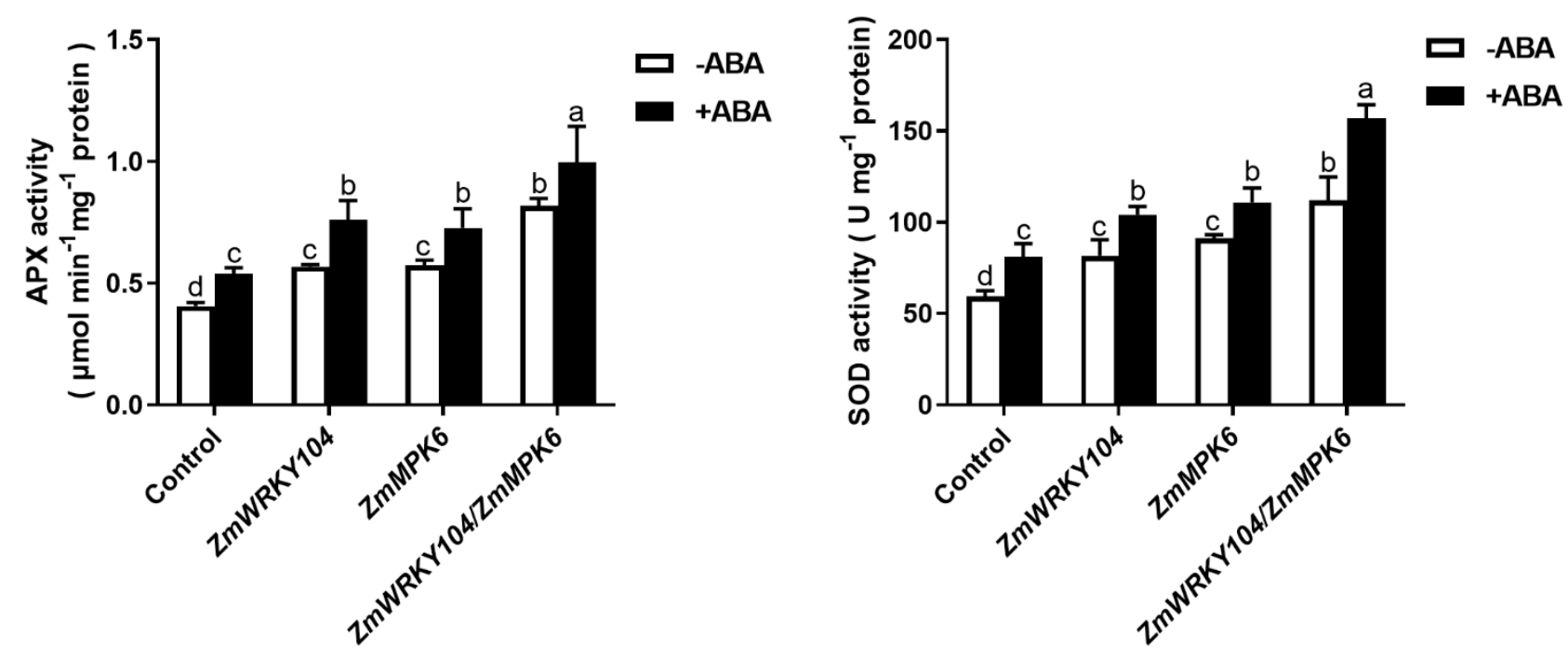

Figure 2. Overexpression of ZmWRKY104 and ZmMPK6 in protoplasts enhances the activities of ABA-induced antioxidant defense enzymes in maize. The activities of APX and SOD in protoplasts transiently expressing ubi: ZmWRKY104-mCherry alone (ZmWRKY104), ubi: ZmMPK6-mCherry alone (ZmMPK6) or ubi: ZmWRKY104-mCherry and ubi: ZmMPK6-mCherry (ZmWRKY104/ZmMPK6) simultaneously in maize mesophyll protoplasts. Culture medium (-ABA) or $10 \mu \mathrm{M}$ ABA (+ABA) were treated with the protoplasts for $10 \mathrm{~min}$. Values are the means $\pm \mathrm{SE}$ of three different experiments. According to Duncan's multiple range test, means represented by the same letter did not significantly differ at $p<0.05$. 


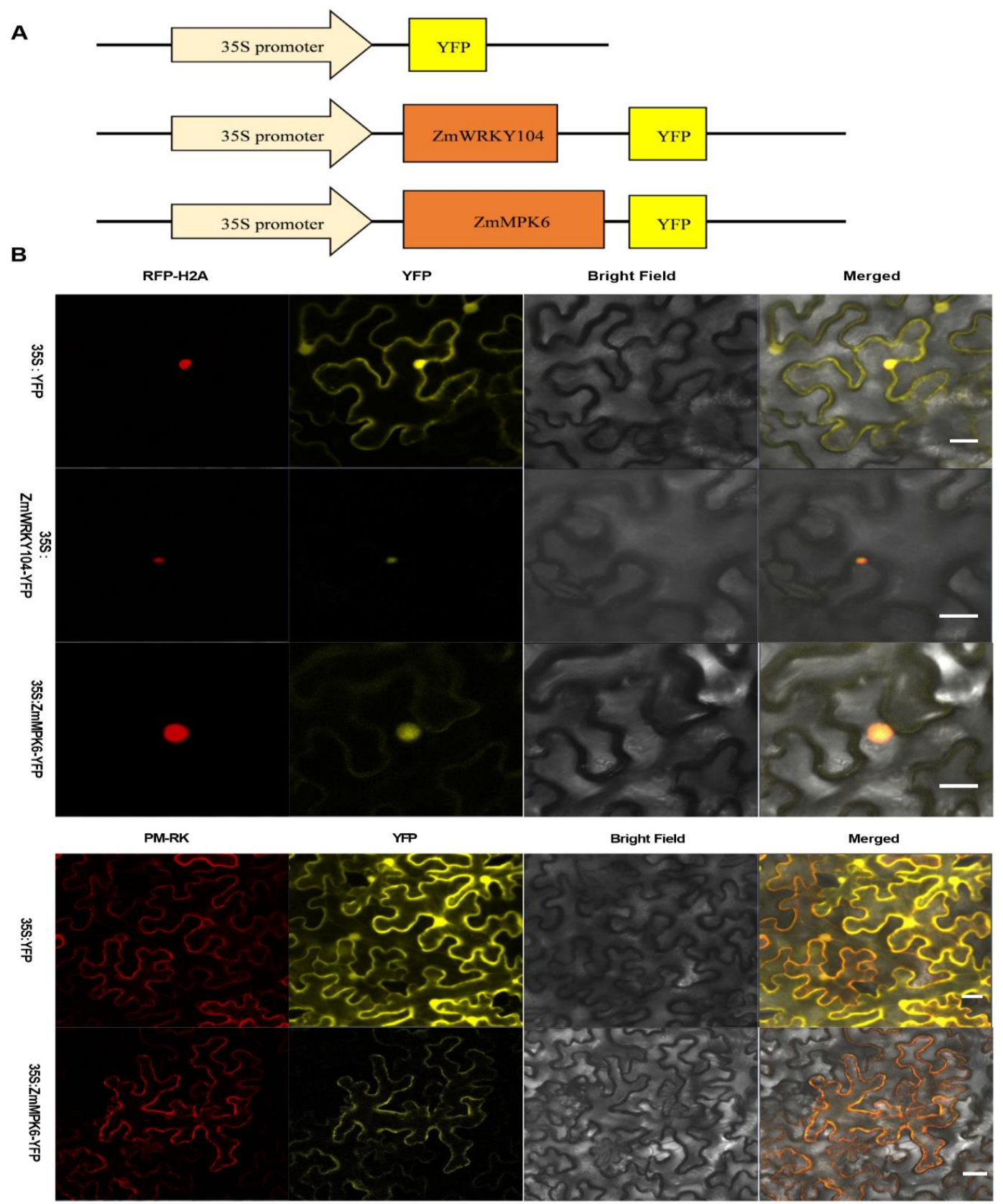

Figure 3. Subcellular localization of ZmWRKY104 and ZmMPK6 in tobacco leaf epidermal cells. (A) Vector diagram of 35S: YFP, 35S: ZmWRKY104-YFP, and 35S: ZmMPK6-YFP; (B) constructs carrying 35S: YFP, 35S: ZmWRKY104-YFP, and 35S: ZmMPK6-YFP were transformed into tobacco leaves by agroinfiltration. Transfected leaves were observed by a confocal laser scanning microscope. Plasma membranes are shown with PM-RK (red). Nuclei are shown with RFP-H2A (red). Experiments were repeated three times with similar results. Scale bars $=20 \mu \mathrm{m}$.

\subsection{ZmMPK6 Interacts with and Phosphorylates ZmWRKY104}

To identify ZmWRKY104 as a target of ZmMPK6, a GST pull-down assay was first used. Full-length ZmWRKY104 fused to GST protein and full-length ZmMPK6 tagged with poly-His. His-tagged ZmMPK6 was maintained on beads with immobilized GSTZmWRKY104 but not with immobilized GST (Figure 4A), which suggests that ZmMPK6 interacted with ZmWRKY104 in vitro. Subsequently, we performed Y2H assays to further identify the interaction in vitro. Full-length ZmWRKY104 was inserted into the pGADT7 (AD) vector, and ZmMPK6 was inserted into the PGBKT7 (BD) vector. Both vectors were then transformed and selected on $\mathrm{SD} / \mathrm{Trp} / \mathrm{Leu} / \mathrm{His} /$ Ade with $\mathrm{X}$ - $\alpha$-galactosidase, 
and the interaction between ZmWRKY104 and ZmMPK6 was observed (Figure 4B). We then performed Co-IP to validate the interaction between ZmWRKY104 and ZmMPK6 in Nicotiana benthamiana leaves. Proteins were immunoprecipitated with protein-A/G agarose beads and analyzed by immunoassay using anti-Myc and anti-Flag antibodies. Immunoblot (IB) analyses using an anti-Myc antibody showed an interaction between Flag-ZmWRKY104 and Myc-ZmMPK6 (Figure 4C). An LCI assay in tobacco leaves was then performed to further confirm the interaction between ZmWRKY104 and ZmMPK6, and the results showed that the ZmWRKY104 protein could interact with the ZmMPK6 protein (Figure 4D). Thus, the above results indicated that ZmMPK6 interacts with ZmWRKY104.

A

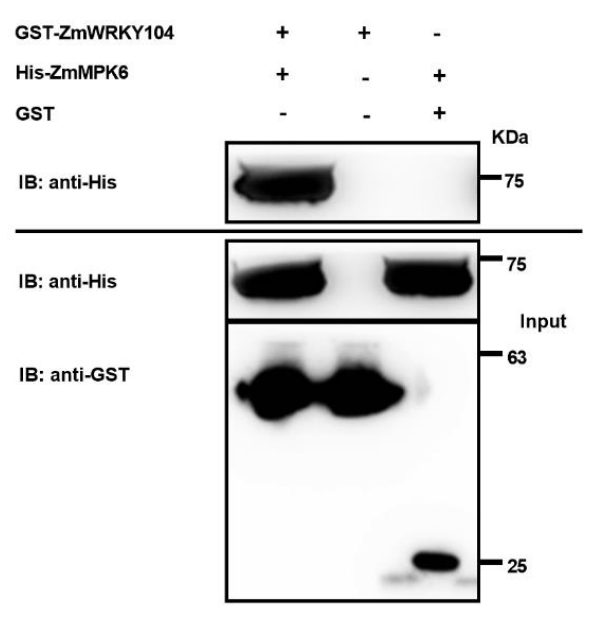

B

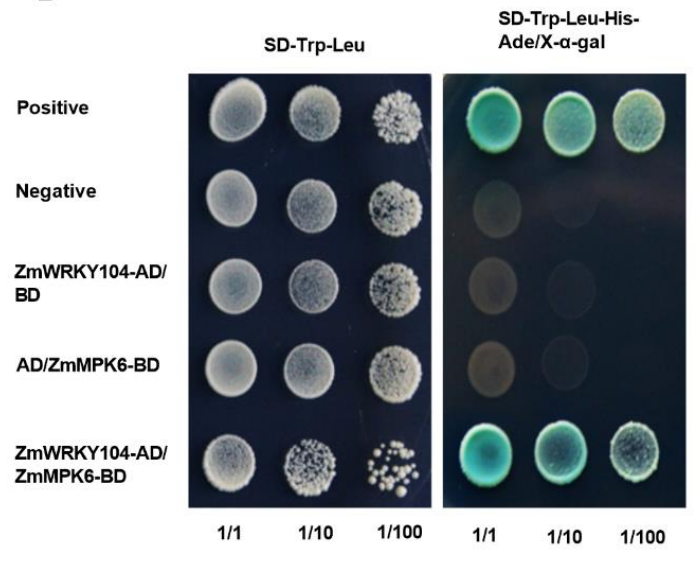

C

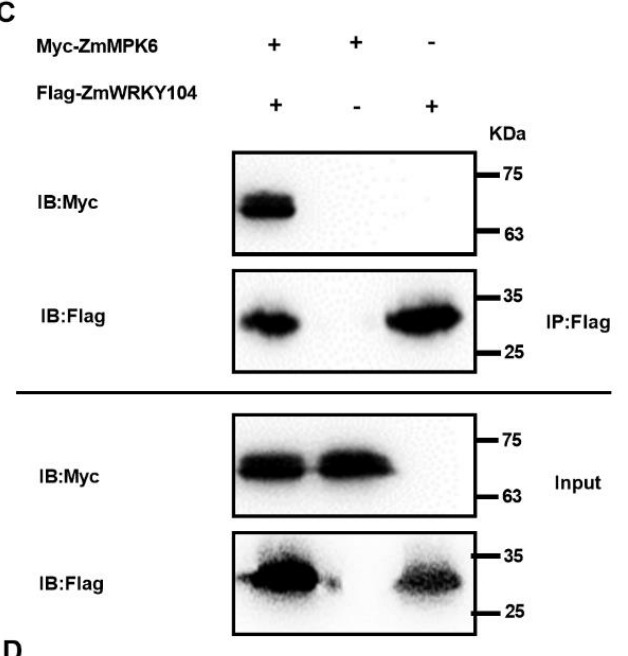

D

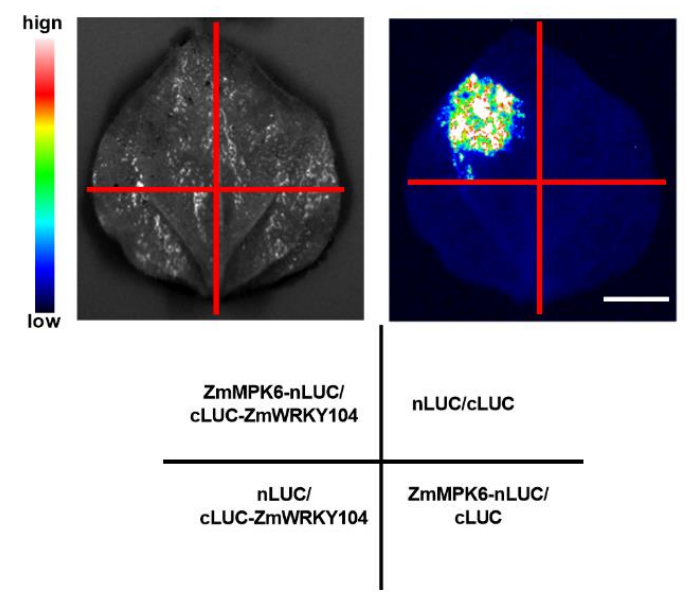

Figure 4. The interaction between ZmWRKY104 and ZmMPK6. (A) Pull-down assay. GST or GST-ZmWRKY104 fusion protein was incubated with His-ZmMPK6 in GST beads. His-ZmMPK6 was detected using an antibody of anti-His by Western blot. GST-ZmWRKY104 and GST were detected using an anti-GST antibody by Western blot. Molecular masses are marked on the right; (B) $\mathrm{Y} 2 \mathrm{H}$ assay. $\mathrm{AD}$-vector and $\mathrm{BD}$-vector were $\mathrm{Y} 2 \mathrm{H}$ vectors with no insert. To test the interaction, the medium of SE-Trp-Leu-His-Ade/X- $\alpha$-gal was used. pGBKT7-53/pGADT7-T was used as a positive control, and pGBKT7lam/pGADT7-T was used as a negative control; (C) Co-IP assay. 35S:Myc-ZmMPK6 and 35S:Flag-ZmWRKY104 were co-expressed in four-week-old Nicotiana benthamiana leaves. Proteins were immunoprecipitated (IP) with Flag antibody and analyzed by immunoblot (IB) using anti-Flag and anti-Myc antibodies. Molecular masses are marked on the right; (D) LCI assay. ZmMPK6 was fused to nLUC, and ZmWRKY104 was fused to cLUC. ZmMPK6-nLUC and cLUC-ZmWRKY104 were then co-expressed in Nicotiana benthamiana leaves. nLUC/cLUC-ZmWRKY104, ZmMPK6-nLUC/cLUC, and nLUC/cLUC were used as negative controls. Luciferase signals were captured using the Tanon 5200 image system. Scale bar $=1 \mathrm{~cm}$. All experiments were repeated at least three times with similar results. 
To examine which region(s) of ZmWRKY104 mediates its binding to ZmMPK6, we generated two truncations, ZmWRKY1041-85AA and ZmWRKY10486-266AA (Figure S5A), and tested these truncations both in vitro and in vivo through GST pull-down and LCI assays. As shown in Figure S5B,C, the interaction region was located at the 1-85 AA region at the $\mathrm{N}$-terminus of ZmWRKY104. To verify that ZmMPK6 phosphorylates ZmWRKY104, we performed an in vitro gel kinase assay. Results showed that ZmMPK6 has autophosphorylation and substrate phosphorylation activity and can phosphorylate myelin basic protein (MBP) and ZmWRKY104 (Figure 5A). Liquid chromatography-tandem mass spectrometry (LC-MS/MS) analysis results showed that Threonine-59 (Thr-59, T59) was the major phosphorylation site of ZmWRKY104 by ZmMPK6 (Figure 5B).

\section{A}

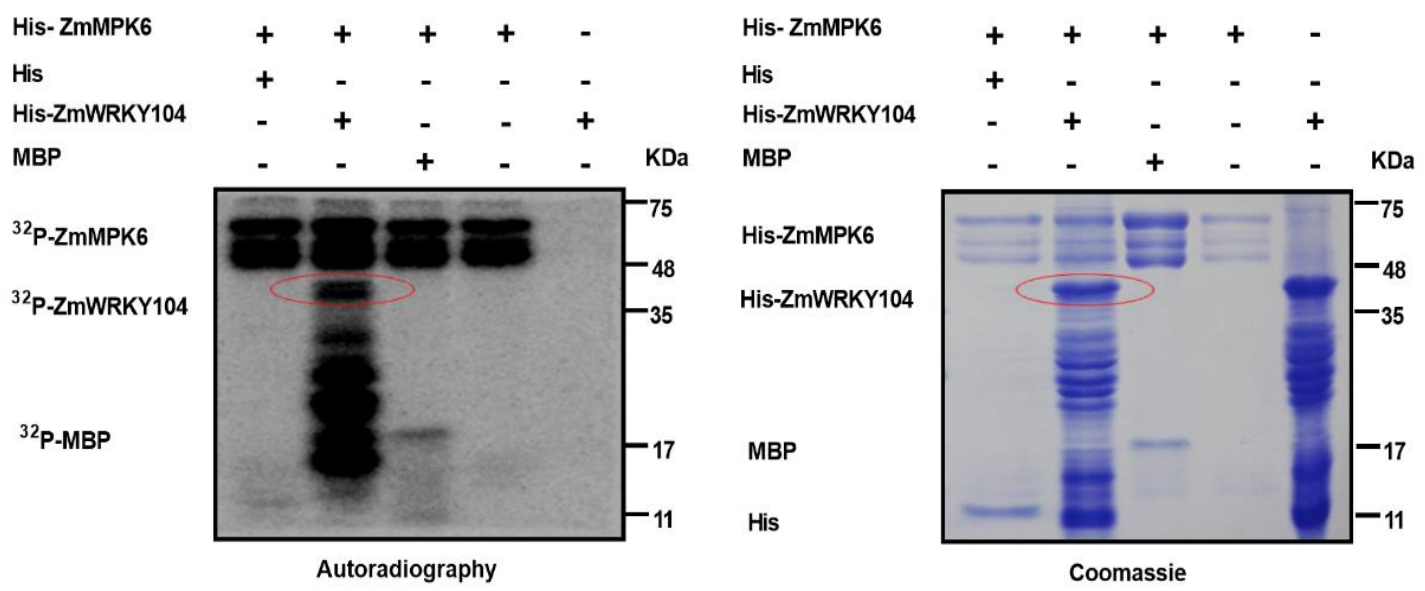

B

\begin{tabular}{ccc}
\hline Peptide & Identified Site & Protein Coverage \\
\hline LAAALDAILADRSPTPR & T59 & $92.9 \%$ \\
\hline
\end{tabular}

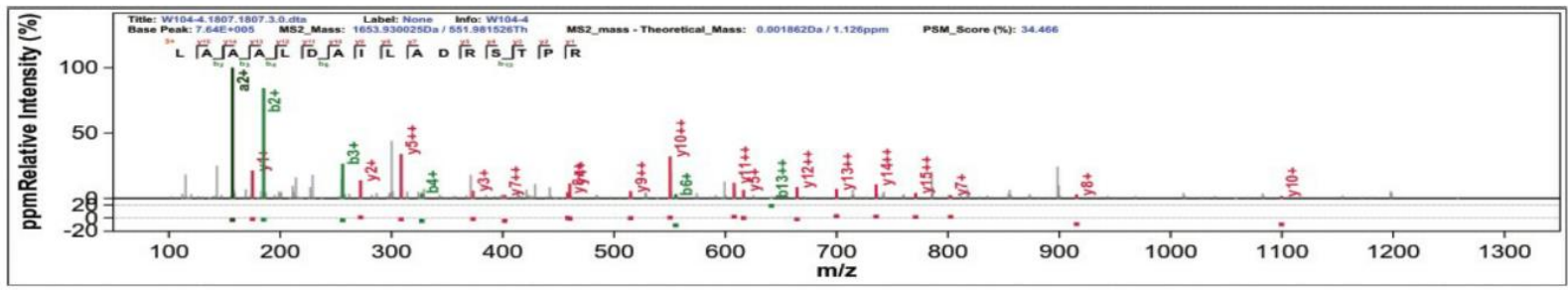

Figure 5. ZmMPK6 phosphorylates ZmWRKY104 in vitro. (A) ZmWRKY104 can be phosphorylated by ZmMPK6 in vitro. His-ZmMPK6, His-ZmWRKY104, and His proteins were expressed in E. coli. In vitro kinase assays were performed using the purified proteins. The substrate of His-ZmWRKY104 or maltose binding protein (MBP) was used for an in-gel kinase assay. Images show autoradiography (left panel) and the corresponding Coomassie staining (right panel). Molecular mass markers in kilodaltons are shown on the right. Experiments were repeated at least three times with similar results. (B) Phosphorylation site(s) analysis of ZmWRKY104 by LC-MS/MS.

\subsection{ZmMPK6 Phosphorylation of ZmWRKY104 Plays a Key Role in ABA-Induced Antioxidant Defense}

To identify whether the Thr-59 site is the major site of ZmWRKY104, we mutated the T59 site of ZmWRKY104 to non-phosphorylated alanine (T59A) and simulated phosphorylated state aspartic acid (T59D) for an in vitro gel kinase assay. Results showed that ZmWRKY104 ${ }^{\mathrm{T} 59 \mathrm{~A}}$ could be weakly phosphorylated by ZmMPK6, indicating that the Thr-59 site of the ZmWRKY104 protein blocked most of the phosphorylation of ZmWRKY104 by 
ZmMPK6 (Figure S6). To explore the role of the Thr-59 site of ZmWRKY104 in ABA-induced antioxidant defense, the maize transient expression system of protoplasts was used to test its effect on the activities of APX and SOD. As shown in Figure 6, compared with the control, transient expression of ZmWRKY104 ${ }^{T 59 A}$ alone did not significantly influence the activities, whereas transient expression of ZmWRKY $104^{T 59 D}$ alone significantly increased the activities. The activities in ZmWRKY104 ${ }^{\mathrm{T} 59 \mathrm{~A}} / \mathrm{ZmMPK6}$-co-expressed protoplasts were equal to those found in protoplasts expressing ZmMPK6 alone. Compared with the control, co-expression of ZmWRKY104 ${ }^{T 59 D} / \mathrm{ZmMPK6}$ significantly increased the activities, and ABA treatment further increased these activities (Figure 6). These data clearly suggest that the phosphorylation of ZmWRKY104 at Thr-59 by ZmMPK6 plays a key role in ABA-induced antioxidant defense.
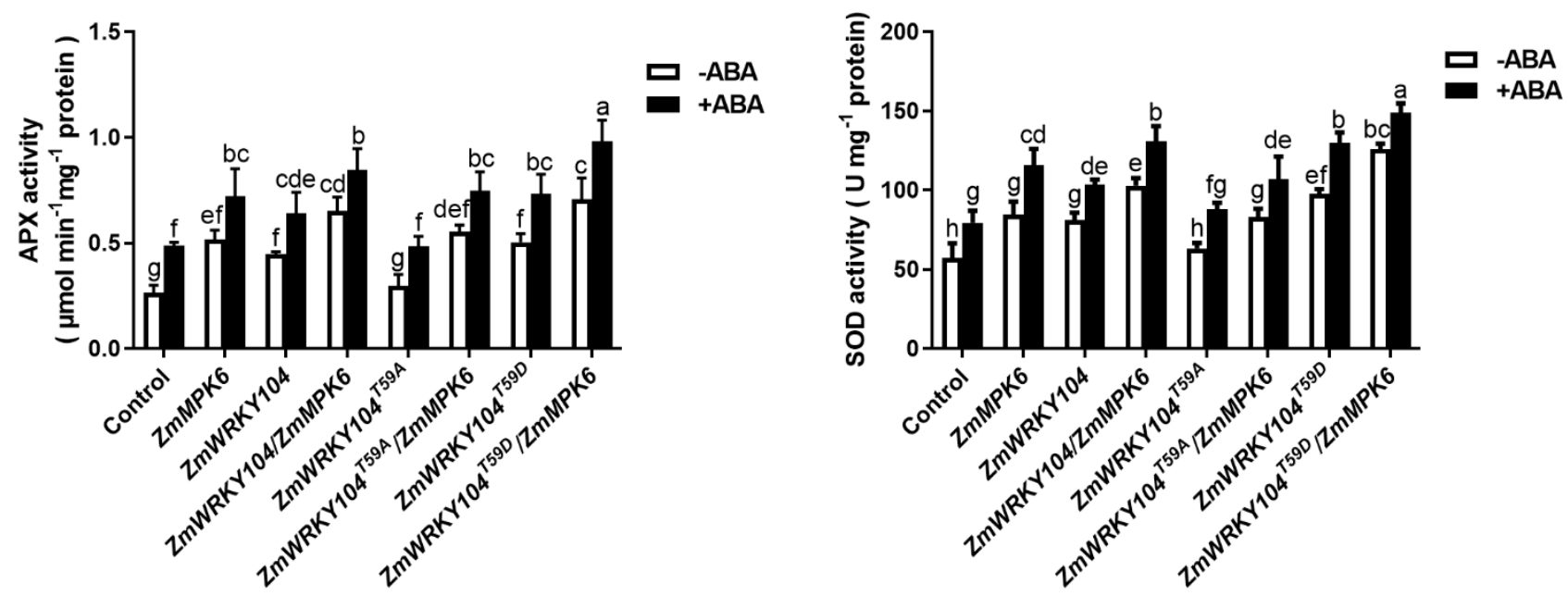

Figure 6. The effects of the Thr-59 site of ZmWRKY104 in ABA-induced antioxidant defense. Ubi: ZmWRKY104mCherry alone (ZmWRKY104), ubi: ZmMPK6-mCherry alone (ZmMPK6) ubi: ZmWRKY104 ${ }^{\text {T59A }}$-mCherry alone (ZmWRKY104 ${ }^{T 59 A}$ ), ubi: ZmWRKY104 ${ }^{T 59 D}$-mCherry alone (ZmWRKY104 $\left.{ }^{T 59 D}\right)$; ubi: ZmWRKY104-mCherry and ubi: ZmMPK6-mCherry (ZmWRKY104/ZmMPK6) simultaneously, ubi: ZmWRKY104 ${ }^{\mathrm{T} 59 A}$-mCherry and ubi: ZmMPK6mCherry (ZmWRKY104 $\left.{ }^{T 59 A} / Z m M P K 6\right)$ simultaneously, and ubi: ZmWRKY104 ${ }^{T 59 D}$-mCherry and ubi: ZmMPK6-mCherry (ZmWRKY104 ${ }^{\mathrm{T} 9 \mathrm{D}} / \mathrm{ZmMPK6}$ ) simultaneously were transiently expressing in protoplasts. Culture medium (-ABA) or 10 $\mu \mathrm{M}$ ABA (+ABA) were treated with the protoplasts for $10 \mathrm{~min}$. Values are the means $\pm \mathrm{SE}$ of three different experiments. According to Duncan's multiple range test, means represented by the same letter did not significantly differ at $p<0.05$.

\subsection{ZmWRKY104 Overexpression Enhances Drought Tolerance in Transgenic Maize Plants}

We used PEG6000 to imitate drought stress to investigate the expression of ZmWRKY104 in maize plants. Results revealed that ZmWRKY104 was powerfully induced at 15 min by PEG (Figure 7A). The above results indicate that $Z m W R K Y 104$ was induced by drought stress.

To further understand the part of ZmWRKY104 in drought stress, we generated transgenic maize plants under the control of the ubiquitin promoter via Agrobacterium tumefaciens-mediated transformation. We selected two independent lines (\#15 and \#17; Figure S7) that exhibited similar ZmWRKY104 gene and protein levels for further analysis. Compared with wild-type (WT) lines, ZmWRKY104-OE transgenic lines displayed no morphological changes under normal growth conditions (Figure 7B). After natural drought, the wild-type lines were restricted, and the leaves were curled and yellow, while the ZmWRKY104-OE transgenic lines grew better, and the leaves were stretched; after rewatering, the leaves of the ZmWRKY104-OE transgenic lines spread out evenly, while the wild-type lines only partially survived and could not fully restore the flat state (Figure 7B). Under drought stress, compared with the WT lines, the ZmWRKY104-OE transgenic lines had higher survival rates (Figure 7C), leaf water retention capacity (Figure 7D), and activity of antioxidant defense enzymes (Figure 7E). In addition, the electrolyte leakage rate (Figure 7F) and malondialdehyde (MDA) content (Figure 7H) of the ZmWRKY104-OE transgenic lines 
were lower than those of the wild-type lines; the relative water content (Figure 7G) and accumulated proline content (Figure 7I) were higher than those of the wild-type lines. The above results indicate that ZmWRKY104 enhances drought stress tolerance.
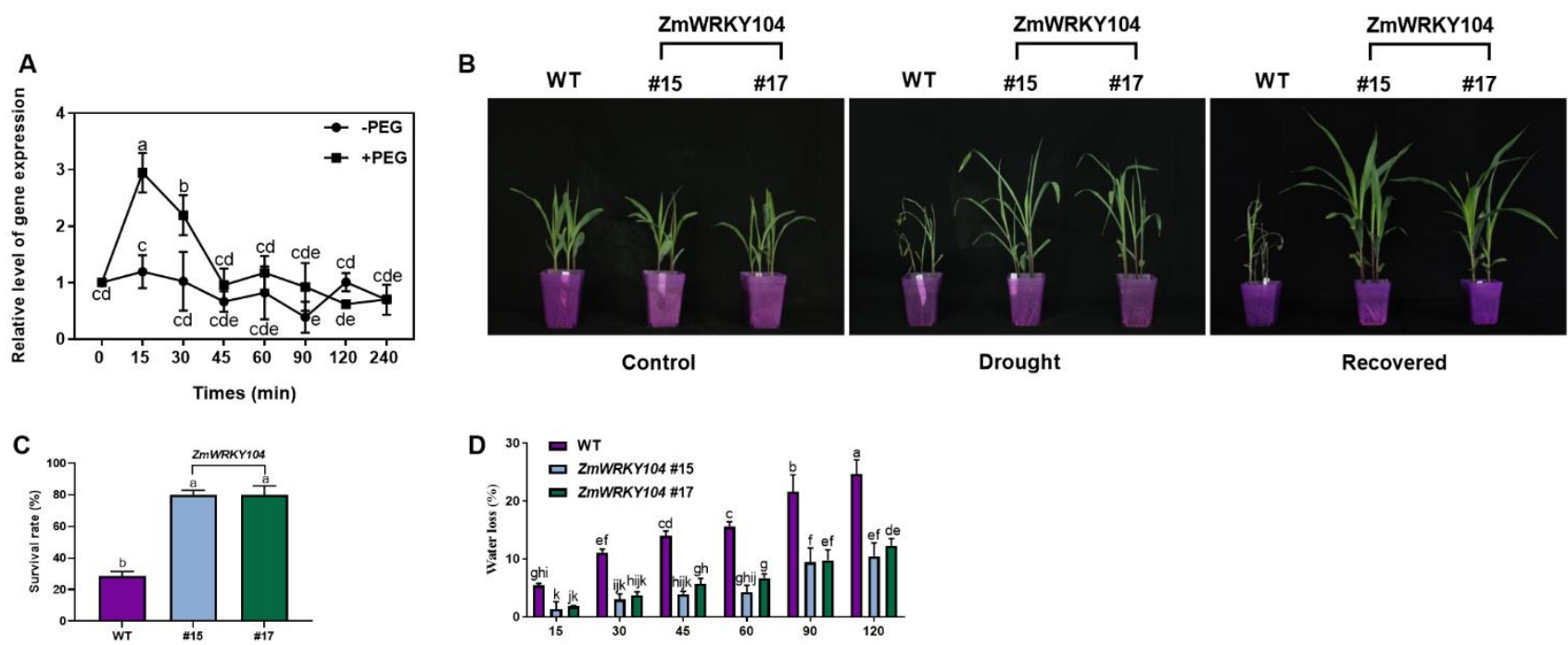

E
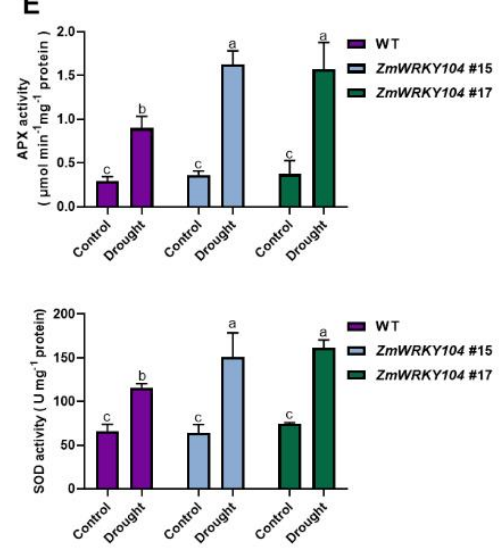

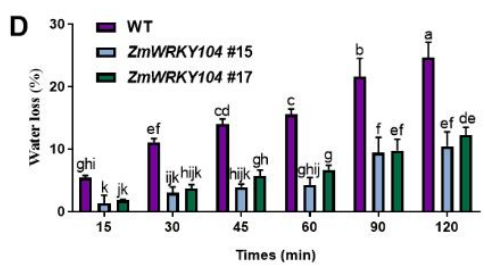

$\mathbf{F}$

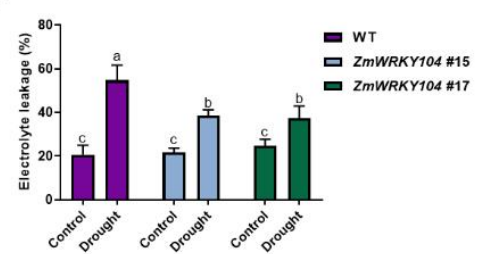

$\mathrm{H}$

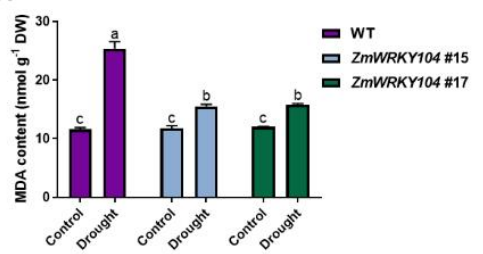

G

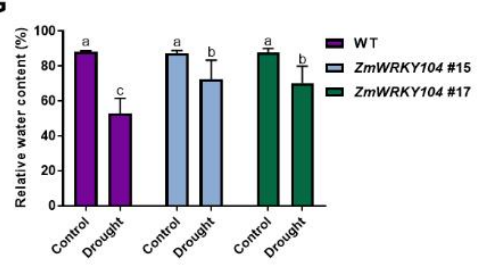

I

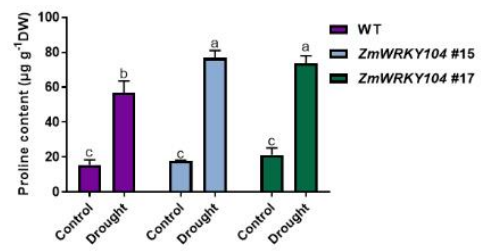

Figure 7. The overexpression of ZmWRKY104 confers drought tolerance in transgenic maize plants. (A) PEG induces the upregulation of ZmWRKY104 expression in the leaves of maize plants. Expression analysis of ZmWRKY104 in leaves of maize plants exposed to $10 \%(w / v)$ polyethylene glycol (PEG6000) treatments for various times as indicated. The expression level relative to ZmActin was analyzed by qRT-PCR. (B) Phenotype of ZmWRKY104 in transgenic maize. Seedlings were subjected to native drought conditions after the three-leaf stage (water was withheld) for 10 days and then rewatered using normal protocols for 3 days. During the experiment, control plants were watered every day. The drought test was repeated 3 times, at least 35 plants were used for each individual line, and a representative picture was displayed. Scale bars $=7 \mathrm{~cm}$. (C) Survival rate of maize plants after rewatering for 3 days. (D) Analysis of water retention in ZmWRKY104-OE transgenic lines. Maize leaves isolated from transgenic and WT seedlings were positioned for $2 \mathrm{~h}$ at $25^{\circ} \mathrm{C}$, and the percentage of water loss at different time points was recorded. (E) Analysis of the activities of APX and SOD of ZmWRKY104-OE transgenic lines under drought stress. The activities were testing of the seedling's leaves treated with Control (distilled water) or drought. (F) Electrolyte leakage; $(\mathbf{G})$ the relative water content, $(\mathbf{H})$ The MDA contents and (I) The proline contents were determined under drought stress and in normal-watered control plants in the leaves of ZmWRKY104-OE transgenic plants and WT plants. Values are the means \pm SE of three different experiments. According to Duncan's multiple range test, means represented by the same letter did not significantly differ at $p<0.05$.

\section{Discussion}

TFs play key roles in plants, allowing them to adjust to new environmental factors, such as drought. As one of the largest families of TFs in higher plants, WRKY families 
play crucial roles in response to various stresses $[12,14,26]$. More and more evidence show that WRKY protein physically interacts with a variety of proteins, and these proteins play a role in controlling or regulating various processes. MAP kinase is one of the main proteins that interact with WRKY TFs [41,42]. The MAP kinase pathway is related to regulate the activities of WRKY33 to manage plant defense responses [43]. The cascade may act as a molecular hub, integrating different signaling networks of WRKY TFs with various upstream proteins. The fact that most WRKYs are distinguishingly regulated by bacterial pathogens or SA treatment further confirms this, which also activates the MPK3/6 pathway [44]. In this study, we demonstrate that ZmWRKY104, which belongs to group IIa of the WRKY superfamily, is a positive component of the ABA-induced antioxidant defense (Figure 1).

The function of TFs or their related proteins could be regulated by MAPK of its phosphorylation [45]. In defense signaling, some studies have shown that WRKY protein is associated with defense-induced MAPK signaling cascade. The NtMEK2-SIPK/WIPK signaling pathway is involved in regulating the expression of defense genes in tobacco [41]. Here, our data show that ZmMPK6-phosphorylated ZmWRKY104 increases ABA-induced antioxidant defense in maize, and phosphorylation at the Thr59 position plays an important role in this process (Figures 5 and 6). Therefore, the modulation of WRKY protein phosphorylated by MAPK seems to be a common phenomenon for regulating plant defense responses.

Subcellular localization experiments revealed that ZmWRKY104 focuses on the nucleus (Figure 3), which accords with the truth that it acts as a TF, potentially modulating the transcription of defense-related genes. However, how Thr-59 phosphorylation affects the DNA binding of ZmWRKY104 is unclear. In addition, the cascade upstream of ZmMPK6 in ABA signaling in maize also needs to be further explored.

Overexpression of ZmWRKY104 modified the drought tolerance of maize plants (Figure 7), indicating that this gene is a positive regulator of drought resistance. Detailed analysis revealed that ZmWRKY104 overexpression alleviates the degree of oxidative damage in response to drought in maize. First, ZmWRKY104 overexpression reduced the electrolyte leakage, which is an immediate stress marker of damage in cell membrane under drought conditions [46]. Second, ZmWRKY104 overexpression lines accumulated higher proline than WT plants. The accumulation of high levels of proline was beneficial to plant resistance to environmental stress [47]. Third, ZmWRKY104 overexpression lines possessed lower MDA contents, and MDA is typically used as an important indicator of oxidative damage under environmental stresses [48]. Fourth, ZmWRKY104 overexpression lines had higher relative water content than WT plants, which is necessary for plants to survive under drought conditions. Furthermore, the alleviation of oxidative damage might be caused by enhanced antioxidant defense.

In summary, our data show that ZmWRKY104 interacts with and is phosphorylated by ZmMPK6, Thr-59 phosphorylation is critical to the role of ZmWRKY104 in ABA-induced antioxidant defense, and overexpression of ZmWRKY104 enhances drought tolerance and alleviates drought-induced oxidative damage.

\section{Materials and Methods}

\subsection{Plant Materials and Treatments}

In this study, we use maize (Zea mays L., cv. B73) and tobacco (Nicotiana benthamiana) seeds. The maize seed was sown in trays placed in an environmental chamber with a photosynthetically active radiation $\left(200 \mu \mathrm{mol} \mathrm{m}{ }^{-2} \mathrm{~s}^{-1}\right)$, a temperature $\left(22-28^{\circ} \mathrm{C}\right)$, and a $14 \mathrm{~h} / 10 \mathrm{~h}$ (day/night) photoperiod and watered every 2 days [49].

Maize plants were grown under dark conditions at $25^{\circ} \mathrm{C}$, and the second leaf was fully extended to extract protoplasts. We cut the plant from the base of the stem and put it in distilled water for $2 \mathrm{~h}$ to relieve the wound pressure. After this treatment, we put the cut end of the stem into a beaker wrapped in aluminum foil, which contained a solution of $100 \mu \mathrm{M}$ ABA and 10\% (w/v) polyethylene glycol (PEG6000) solution. Plants immersed in distilled water for the entire period under the same conditions served as controls. After the 
treatment, subsequent leaves of the separated maize plants were sampled and immediately frozen in liquid nitrogen.

Tobacco seeds were sown in a soil pot with a photosynthetically active radiation $\left(120 \mu \mathrm{mol} \mathrm{m}{ }^{-2} \mathrm{~s}^{-1}\right)$, a temperature $\left(23^{\circ} \mathrm{C}\right)$, and a photoperiod of $16 \mathrm{~h} / 8 \mathrm{~h}$ (day/night) and watered every two days. After 4 weeks of growth, we use the plants for subsequent experiments.

\subsection{GST Pull-Down Assay}

GST, GST-ZmWRKY104, and truncated (GST-ZmWRKY1041-85AA and GST-ZmWRKY104 ${ }^{86-266 A A}$ ) fusion proteins were maintained, immobilized, on Magnet GST particles (Promega, Madison, USA) and then incubated with His-tagged ZmMPK6 in binding buffer $\left(10 \mathrm{mM} \mathrm{KCl}, 4.2 \mathrm{mM} \mathrm{Na}_{2} \mathrm{HPO}_{4}, 140 \mathrm{mM} \mathrm{NaCl}, 2 \mathrm{mM} \mathrm{KH} \mathrm{PO}_{4}\right.$, and 10\% bovine serum albumin, $\mathrm{pH}$ 7.2) with gentle shaking for $2 \mathrm{~h}$ at $4{ }^{\circ} \mathrm{C}$. Then, washing buffer (140 mM NaCl, $4.2 \mathrm{mM} \mathrm{Na}_{2} \mathrm{HPO}_{4}, 2 \mathrm{mM} \mathrm{KH} \mathrm{PO}_{4}$, and $10 \mathrm{mM} \mathrm{KCl}, \mathrm{pH}$ 7.2) wash the beads more than 3 times, and use the $1 \times$ SDS loading buffer (50 mM Tris-HCl, 2\% SDS, $0.1 \%$ bromophenol blue, $10 \%$ glycerol, and $10 \mathrm{mM}$ dithiothreitol (DTT)) to elute the pulled down proteins by boiling, use the $12 \%$ SDS-PAGE gels to separate them, use an anti-GST antibody (Abmart, Shanghai, China) or anti-His antibody (Abmart) followed by a goat anti-mouse IgG HRP-conjugated secondary antibody (Abmart).to analyze.

\subsection{Yeast Two-Hybrid (Y2H) Assay}

According to the yeast protocol handbook (Clontech, Shiga, Japan), Y2H assays were performed. ZmWRKY104 was cloned into pGADT7 using EcoRI/BamHI sites, ZmMPK6 was cloned into pGBKT7 using BamH/PstI sites, and Table S1 shows the primers. The prey vector was converted into Y187 of yeast strain, and the bait vector was transformed into Y2HGold of yeast strain using the lithium acetate method. The prey and bait strains were mated and spread on stringent selective medium plates containing $X-\alpha$-gal $\left(40 \mu \mathrm{g} \mathrm{mL}{ }^{-1}\right)$, then incubated for $3-5$ days at $30{ }^{\circ} \mathrm{C}$ and verified every day.

\subsection{Co-Immunoprecipitation (Co-IP) Assay}

ZmWRKY104 or ZmMPK6 were fused to Flag or Myc tags cloned into 1300-221 vectors using KpnI/BamHI sites or BstBI/BamHI sites. Table S1 shows the primers. 35S: MycZmMPK6, 35S: Flag-ZmWRKY104, 35S: Myc-ZmMPK6, and 35S: Flag-ZmWRKY104 were expressed in Nicotiana benthamiana leaves. After incubation for 3 days, proteins were extracted from the leaves with buffer as described previously and centrifuged at $4{ }^{\circ} \mathrm{C}$ at $10,000 \mathrm{~g}$ for $30 \mathrm{~min}$ [6]. The soluble proteins were incubated with an anti-Flag (Abmart) or anti-Myc antibody (Abmart) bound to IP buffer at $4{ }^{\circ} \mathrm{C}$ for $3 \mathrm{~h}$. We washed the beads with immunoprecipitation buffer 3 times and boiled for $8 \mathrm{~min}$ in $1 \times$ SDS buffer to elute proteins. After centrifugation, the supernatant was analyzed with anti-MyC antibody (Abmart) by immunoblotting.

\subsection{Luciferase Complementation Imaging (LCI) Assay}

We fused full-length and truncated sequences of ZmWRKY104 to the pC1300-cLUC vector utilizing KpnI/BamHI sites, and fused full-length sequences of ZmMPK6 to the pC1300-nLUC vector utilizing SacI/BamHI sites. Table S1 shows the primers. Then we transformed them into GV3101 of Agrobacterium tumefaciens strain and incubated under dark conditions at $30^{\circ} \mathrm{C}$ for 2-3 days, and selected the positive clone and incubated in yeast extract broth (YEB) liquid medium for $16 \mathrm{~h}$ at $28^{\circ} \mathrm{C}$ [49]. The bacteria were collected in $2 \mathrm{~mL}$ tubes and centrifuged at $5000 \mathrm{rpm}$ for $5 \mathrm{~min}$ at $25^{\circ} \mathrm{C}$. Then we removed the supernatant and resuspended the bacteria in buffer $\left(10 \mathrm{mM} \mathrm{MgCl}_{2}, 10 \mathrm{mM} \mathrm{MES}, \mathrm{pH} 5.7\right.$, and $100 \mu \mathrm{M}$ acetosyringone) to a final OD600 of 0.5 . After 3 to $5 \mathrm{~h}$, we injected the bacteria into Nicotiana benthamiana leaves, and $72 \mathrm{~h}$ after injection, we sprayed with $1 \mathrm{mM}$ D-Luciferin (Thermo Fisher Scientific, Waltham, MA, USA) at the abaxial sides of the leaves. The leaves were then stored for 30 min in the dark, and we used a camera (Tanon 5200 Multi, Tanon Biomart, Beijing, China) to capture the signal of LUC. 


\subsection{Expression and Purification of Recombinant Proteins}

ZmWRKY104 and its truncated mutants (ZmWRKY104 ${ }^{1-85 A A}$ and ZmWRKY104 ${ }^{86-266 A A}$ ), were amplified, cloned into the vector of $p G E X-4 T-1$ with a GST tag, and expressed in BL21 (DE3) of E. coli strain. The expression of plasmids or the empty vector was induced for $6 \mathrm{~h}$ at $28{ }^{\circ} \mathrm{C}$ with isopropyl $\beta$-D-1-thiogalactopyranoside (IPTG) of $0.2 \mathrm{mM}$ in LB broth on a shaker at $160 \mathrm{rpm}$. Full-length ZmWRKY104, ZmWRKY104 ${ }^{\text {T59A }}$ or ZmWRKY104 ${ }^{\text {T59D }}$ was transformed into BL21 (DE3) of E. coli with the His-tagged expression vector pET-30a, and induced by incubation for $6 \mathrm{~h}$ at $2{ }^{\circ} \mathrm{C}$ with $0.2 \mathrm{mM}$ IPTG. Full-length ZmMPK6 was cloned into the pET-30a vector in frame with the His tag, transformed into BL21 (DE3) of E. coli strain, and induced by incubation for $6 \mathrm{~h}$ at $26^{\circ} \mathrm{C}$ with $0.5 \mathrm{mM}$ IPTG.

\subsection{In Vitro Kinase Assay}

Recombinant His-ZmWRKY104, His-ZmWRKY104 ${ }^{\mathrm{T} 59 \mathrm{~A}}$ or His-ZmWRKY104 ${ }^{\mathrm{T} 59 \mathrm{D}}$ was incubated with $0.01 \mathrm{mg} \mathrm{mL}^{-1} \mathrm{ZmMPK6}$ protein in buffer of phosphorylation $(20 \mathrm{mM}$ $\mathrm{MgCl}_{2}, 25 \mathrm{mM}$ Tris- $\mathrm{HCl}, \mathrm{pH} 7.5,2 \mathrm{mM} \mathrm{MnCl}$, and $1 \mathrm{mM}$ DTT) for $30 \mathrm{~min}$ at $30^{\circ} \mathrm{C}$ in a final volume of $50 \mu \mathrm{L}$ including $1 \mu \mathrm{g}$ of $\mathrm{MBP}, 10 \mu \mathrm{M}$ ATP, and $10 \mu \mathrm{Ci}\left[\gamma_{-}{ }^{32} \mathrm{P}\right] \mathrm{ATP}$. We added SDS sample buffer to stop the reaction and boiled the product for $10 \mathrm{~min}$ in a boiling water bath and resolved by $12 \%$ SDS-PAGE. We removed the unincorporated $\left[\gamma_{-}{ }^{32} \mathrm{P}\right]$ ATP by washing with $5 \%$ trichloroacetic acid $(w / v)$ and $1 \%$ sodium pyrophosphate $(w / v)$ more than three times. The phosphorylated substrates were visualized by autoradiography.

\subsection{Isolation of Total RNA and qRT-PCR Analysis}

The analysis was performed as performed as described previously [7]. The primers are described in detail in Table S1.

\subsection{Vector Construction and In Vitro Synthesis of Double-Stranded RNA}

The construction and synthesis were performed as described previously [7]. The primers are given in Table S1.

\subsection{Protoplast Preparation and Transfection with DNA Constructs or dsRNAs}

The experiments were performed as indicated previously [7]. The transformed protoplasts were then incubated overnight at $25^{\circ} \mathrm{C}$ in the dark in culture solution, treated for 10 min with or without $10 \mu \mathrm{M} \mathrm{ABA}$, and utilized for the subsequent analysis.

\subsection{Site-Directed Mutagenesis}

For the mutation of ZmWRKY104, we utilized the M5 HiPer Site-Directed Mutagenesis Kit (Mei5bio, Beijing, China) based on the manufacturer's instructions. Table S1 shows the DNA oligonucleotides sequences.

\subsection{Subcellular Localization}

Tobacco leaves were transfected with GV3101 of Agrobacterium strain carrying the fusion construct of 35S: ZmWRKY104-YFP, the 35S: ZmMPK6-YFP fusion construct, or 35S: YFP. Yellow fluorescence was observed as indicated previously under a Zeiss LSM710 device [7]. The nucleus was stained with a nuclear marker (RFP-H2A), and the plasma membrane was stained with a membrane marker (PM-RK) [50].

\subsection{Antioxidant Enzyme Assay}

The antioxidant enzyme assays were performed as indicated previously described [51-53].

\subsection{Mass Spectrometry Analysis}

The His-ZmWRKY104 fusion protein was reacted with the His-ZmMPK6 fusion protein in kinase assay buffer in vitro with ATP. Then we enriched the phosphorylated ZmWRKY104 and digested by trypsin followed by mass spectrometry/liquid chromatography tandem mass spectrometry (LC-MS/MS) analysis as indicated previously [7]. The 
results of mass spectrometry were analyzed using the software pFind Studio (http:/ / pfind. ict.ac.cn/software/pLink/index.html, accessed on 23 Jul 2020).

\subsection{Generation of Transgenic Maize Plants}

Insert ZmWRKY104 into the HindIII/KpnI sites of the pCUN-N-HF vector with ubiquitin promoter; Table S1 shows the primers. We used the maize inbred line B73 for genetic transformation. The recombinant vector was introduced into maize by LBA4404 of Agrobacterium tumefaction strain via maize shoot tip transformation [54,55]. Agrobacteria containing the recombinant strain were cultured separately and then mixed in infiltration buffer to a final OD600 between 0.65 and 0.75 . The mixture of Agrobacterium cultures were infiltrated with young maize stems by a vacuum pump ( 0.045 to $0.060 \mathrm{MPa})$ for 3 to $5 \mathrm{~min}$ and then sown in pots containing a 1:1 mixture of organic nutrient soil vermiculite. The pots were placed in an environmental chamber with $22-28^{\circ} \mathrm{C}$, photosynthetically active radiation $\left(200 \mu \mathrm{mol} \mathrm{m}^{-2} \mathrm{~s}^{-1}\right)$, and a $14 \mathrm{~h} / 10 \mathrm{~h}$ (day/night) photoperiod and watered every 3 days. The positive plants were identified by PCR analysis and then transferred to the greenhouse for planting and harvesting, and Table S1 shows the primers. qRT-PCR analyzed the expression of ZmWRKY104 in transgenic lines, and two independent homozygous T2 lines, ZmWRKY104 \#15 and ZmWRKY104 \#17, were selected for subsequent experiments.

\subsection{Drought Tolerance and Oxidative Damage Analysis}

After 4 weeks of growth, seedlings were subjected to native drought conditions (water was withheld) for 10 days and then rewatered using normal protocols for 3 days. The seedling phenotype was photographed, and the survival rate was calculated. The experiments were repeated 3 times, each individual line with more than 35 plants employed in every repeated experiment, and one typical image is shown. The relative water content (RWC), malondialdehyde (MDA) levels, electrolyte leakage, the content of proline (Pro), and APX and SOD activities were measured as previously described [7,56-58].

\subsection{Phylogenetic Analysis}

To construct a phylogenetic tree, the sequences of plant WRKY proteins and MAPK proteins were retrieved from the NCBI database and aligned using the ClustalW program, a neighbor-joining phylogenetic tree of these sequences was then constructed, and bootstrap analysis was performed with 500 iterations using the MEGA7 program [52,59].

\section{Conclusions}

In this study, we identified a WRKY IIa TF, ZmWRKY104, as the substrate protein of ZmMPK6 in maize. Liquid chromatography-tandem mass spectrometry (LC-MS/MS) analysis results showed that threonine-59 (Thr-59, T59) was the major phosphorylation site of ZmWRKY104 by ZmMPK6. Functional analysis revealed that the role of ZmWRKY104 in ABA-induced antioxidant defense depends on ZmMPK6. Genetic analysis showed that overexpression of ZmWRKY104 in maize can improve drought tolerance and alleviate drought-induced oxidative damage in transgenic plants.

Supplementary Materials: The following are available online at https: / www.mdpi.com/article / 10.3390/biology10090893/s1, Figure S1. Multiple alignment of ZmWRKY104 with other orthologs in rice, Arabidopsis. Figure S2. Phylogenetic relationships of ZmWRKY104 with other orthologs in rice, Arabidopsis. Figure S3. Multiple alignment and phylogenetic relationships of ZmMPK6 with other orthologs in rice, Arabidopsis, wheat, cotton. Figure S4. RT-qPCR analysis of ZmWRKY104 expression in different tissues of maize, Figure S5. ZmMPK6 interacts with the truncations of ZmWRKY104, Figure S6. Phosphorylation of ZmWRKY104 ${ }^{\mathrm{T} 59 \mathrm{~A}}$ and ZmWRKY104 ${ }^{\mathrm{T} 59 \mathrm{D}}$ by ZmMPK6 in vitro, Figure S7. Analysis the gene expression and protein level of the ZmWRKY104 transgenic lines, Table S1: PCR Primers Used.

Author Contributions: Conceptualization, L.Z. and A.Z.; data curation, L.Z., J.Y., Y.X., Y.S., A.Z.; formal analysis, L.Z., J.Y., Y.S., A.Z.; methodology, L.Z. and Y.X.; investigation, L.Z., J.Y., Y.X., Y.S., 
A.Z.; writing—original draft preparation, L.Z.; writing—review and editing, J.Y. and A.Z.; funding acquisition, A.Z. All authors have read and agreed to the published version of the manuscript.

Funding: This study is supported by grants from the National Natural Science Foundation of China (31871534 and 32001445), the Natural Science Foundation of Jiangsu Province (BK20200557).

Institutional Review Board Statement: Not applicable.

Informed Consent Statement: Not applicable.

Data Availability Statement: Not applicable.

Conflicts of Interest: The authors declare that they have no conflicts of interest.

\section{References}

1. Sade, N.; Del Mar Rubio-Wilhelmi, M.; Umnajkitikorn, K.; Blumwald, E. Stress-induced senescence and plant tolerance to abiotic stress. J. Exp. Bot. 2018, 69, 845-853. [CrossRef] [PubMed]

2. Gong, Z.; Xiong, L.; Shi, H.; Yang, S.; Herrera-Estrella, L.R.; Xu, G.; Chao, D.Y.; Li, J.; Wang, P.Y.; Qin, F.; et al. Plant abiotic stress response and nutrient use efficiency. Sci. China Life Sci. 2020, 63, 635-674. [CrossRef] [PubMed]

3. de Zelicourt, A.; Colcombet, J.; Hirt, H. The Role of MAPK Modules and ABA during Abiotic Stress Signaling. Trends Plant Sci. 2016, 21, 677-685. [CrossRef] [PubMed]

4. Gupta, S.; Dong, Y.; Dijkwel, P.P.; Mueller-Roeber, B.; Gechev, T.S. Genome-Wide Analysis of ROS Antioxidant Genes in Resurrection Species Suggest an Involvement of Distinct ROS Detoxification Systems during Desiccation. Int. J. Mol. Sci. 2019, 20, 3101. [CrossRef] [PubMed]

5. Ma, F.; Lu, R.; Liu, H.; Shi, B.; Zhang, J.; Tan, M.; Zhang, A.; Jiang, M. Nitric oxide-activated calcium/calmodulin-dependent protein kinase regulates the abscisic acid-induced antioxidant defence in maize. J. Exp. Bot. 2012, 63, 4835-4847. [CrossRef] [PubMed]

6. Zhang, A.; Jiang, M.; Zhang, J.; Tan, M.; Hu, X. Mitogen-activated protein kinase is involved in abscisic acid-induced antioxidant defense and acts downstream of reactive oxygen species production in leaves of maize plants. Plant Physiol. 2006, 141, 475-487. [CrossRef] [PubMed]

7. Zhu, Y.; Yan, J.; Liu, W.; Liu, L.; Sheng, Y.; Sun, Y.; Li, Y.; Scheller, H.V.; Jiang, M.; Hou, X.; et al. Phosphorylation of a NAC Transcription Factor by a Calcium/Calmodulin-Dependent Protein Kinase Regulates Abscisic Acid-Induced Antioxidant Defense in Maize. Plant Physiol. 2016, 171, 1651-1664. [CrossRef] [PubMed]

8. Khaleghnezhad, V.; Yousefi, A.R.; Tavakoli, A.; Farajmand, B.; Mastinu, A. Concentrations-dependent effect of exogenous abscisic acid on photosynthesis, growth and phenolic content of Dracocephalum moldavica L. under drought stress. Planta 2021, $253,127$. [CrossRef]

9. Umezawa, T. Systems biology approaches to abscisic acid signaling. J. Plant Res. 2011, 124, 539-548. [CrossRef]

10. Meng, X.; Zhang, S. MAPK cascades in plant disease resistance signaling. Annu. Rev. Phytopathol. 2013, 51, 245-266. [CrossRef]

11. Hamel, L.P.; Nicole, M.C.; Sritubtim, S.; Morency, M.J.; Ellis, M.; Ehlting, J.; Beaudoin, N.; Barbazuk, B.; Klessig, D.; Lee, J.; et al. Ancient signals: Comparative genomics of plant MAPK and MAPKK gene families. Trends Plant Sci. 2006, 11, 192-198. [CrossRef]

12. Wang, M.; Zhang, Y.; Wang, J.; Wu, X.; Guo, X. A novel MAP kinase gene in cotton (Gossypium hirsutum L.), GhMAPK, is involved in response to diverse environmental stresses. J. Biochem. Mol. Biol. 2007, 40, 325-332. [CrossRef] [PubMed]

13. Zong, X.J.; Li, D.P.; Gu, L.K.; Li, D.Q.; Liu, L.X.; Hu, X.L. Abscisic acid and hydrogen peroxide induce a novel maize group C MAP kinase gene, ZmMPK7, which is responsible for the removal of reactive oxygen species. Planta 2009, 229, 485-495. [CrossRef] [PubMed]

14. Shi, J.; An, H.L.; Zhang, L.; Gao, Z.; Guo, X.Q. GhMPK7, a novel multiple stress-responsive cotton group C MAPK gene, has a role in broad spectrum disease resistance and plant development. Plant Mol. Biol. 2010, 74, 1-17. [CrossRef] [PubMed]

15. Dóczi, R.; Brader, G.; Pettkó-Szandtner, A.; Rajh, I.; Djamei, A.; Pitzschke, A.; Teige, M.; Hirt, H. The Arabidopsis mitogenactivated protein kinase kinase MKK3 is upstream of group $\mathrm{C}$ mitogen-activated protein kinases and participates in pathogen signaling. Plant Cell 2007, 19, 3266-3279. [CrossRef] [PubMed]

16. Zhang, L.; Xi, D.; Luo, L.; Meng, F.; Li, Y.; Wu, C.A.; Guo, X. Cotton GhMPK2 is involved in multiple signaling pathways and mediates defense responses to pathogen infection and oxidative stress. FEBS J. 2011, 278, 1367-1378. [CrossRef] [PubMed]

17. Walia, A.; Lee, J.S.; Wasteneys, G.; Ellis, B. Arabidopsis mitogen-activated protein kinase MPK18 mediates cortical microtubule functions in plant cells. Plant J. 2009, 59, 565-575. [CrossRef] [PubMed]

18. Pan, J.; Zhang, M.; Kong, X.; Xing, X.; Liu, Y.; Zhou, Y.; Liu, Y.; Sun, L.; Li, D. ZmMPK17, a novel maize group D MAP kinase gene, is involved in multiple stress responses. Planta 2012, 235, 661-676. [CrossRef] [PubMed]

19. Lalle, M.; Visconti, S.; Marra, M.; Camoni, L.; Velasco, R.; Aducci, P. ZmMPK6, a novel maize MAP kinase that interacts with 14-3-3 proteins. Plant Mol. Biol. 2005, 59, 713-722. [CrossRef] [PubMed]

20. Jiang, J.; Ma, S.; Ye, N.; Jiang, M.; Cao, J.; Zhang, J. WRKY transcription factors in plant responses to stresses. J. Integr. Plant Biol. 2017, 59, 86-101. [CrossRef] 
21. Xie, T.; Chen, C.; Li, C.; Liu, J.; Liu, C.; He, Y. Genome-wide investigation of WRKY gene family in pineapple: Evolution and expression profiles during development and stress. BMC Genom. 2018, 19, 490. [CrossRef] [PubMed]

22. Rushton, P.J.; Somssich, I.E.; Ringler, P.; Shen, Q.J. WRKY transcription factors. Trends Plant Sci. 2010, 15, 247-258. [CrossRef] [PubMed]

23. Wei, K.F.; Chen, J.; Chen, Y.F.; Wu, L.J.; Xie, D.X. Molecular phylogenetic and expression analysis of the complete WRKY transcription factor family in maize. DNA Res. 2012, 19, 153-164. [CrossRef] [PubMed]

24. Jiang, Y.; Liang, G.; Yu, D. Activated expression of WRKY57 confers drought tolerance in Arabidopsis. Mol Plant 2012, 5, 1375-1388. [CrossRef] [PubMed]

25. Ding, Z.J.; Yan, J.Y.; Li, C.X.; Li, G.X.; Wu, Y.R.; Zheng, S.J. Transcription factor WRKY46 modulates the development of Arabidopsis lateral roots in osmotic/salt stress conditions via regulation of ABA signaling and auxin homeostasis. Plant J. 2015, 84, 56-69. [CrossRef]

26. He, G.H.; Xu, J.Y.; Wang, Y.X.; Liu, J.M.; Li, P.S.; Chen, M.; Ma, Y.Z.; Xu, Z.S. Drought-responsive WRKY transcription factor genes TaWRKY1 and TaWRKY33 from wheat confer drought and/or heat resistance in Arabidopsis. BMC Plant Biol. 2016, 16, 116. [CrossRef] [PubMed]

27. Chen, J.; Nolan, T.M.; Ye, H.; Zhang, M.; Tong, H.; Xin, P.; Chu, J.; Chu, C.; Li, Z.; Yin, Y. Arabidopsis WRKY46, WRKY54, and WRKY70 Transcription Factors Are Involved in Brassinosteroid-Regulated Plant Growth and Drought Responses. Plant Cell 2017, 29, 1425-1439. [CrossRef] [PubMed]

28. Shi, W.Y.; Du, Y.T.; Ma, J.; Min, D.H.; Jin, L.G.; Chen, J.; Chen, M.; Zhou, Y.B.; Ma, Y.Z.; Xu, Z.S.; et al. The WRKY Transcription Factor GmWRKY12 Confers Drought and Salt Tolerance in Soybean. Int. J. Mol. Sci. 2018, 19, 4087. [CrossRef] [PubMed]

29. Peng, Y.; Bartley, L.E.; Canlas, P.; Ronald, P.C. OsWRKY IIa Transcription Factors Modulate Rice Innate Immunity. Rice 2010, 3, 36-42. [CrossRef]

30. Eckey, C.; Korell, M.; Leib, K.; Biedenkopf, D.; Jansen, C.; Langen, G.; Kogel, K.H. Identification of powdery mildew-induced barley genes by cDNA-AFLP: Functional assessment of an early expressed MAP kinase. Plant Mol. Biol. 2004, 55, 1-15. [CrossRef]

31. Shen, Q.H.; Saijo, Y.; Mauch, S.; Biskup, C.; Bieri, S.; Keller, B.; Seki, H.; Ulker, B.; Somssich, I.E.; Schulze-Lefert, P. Nuclear activity of MLA immune receptors links isolate-specific and basal disease-resistance responses. Science 2007, 315, 1098-1103. [CrossRef] [PubMed]

32. Chen, H.; Lai, Z.; Shi, J.; Xiao, Y.; Chen, Z.; Xu, X. Roles of arabidopsis WRKY18, WRKY40 and WRKY60 transcription factors in plant responses to abscisic acid and abiotic stress. BMC Plant Biol. 2010, 10, 281. [CrossRef] [PubMed]

33. Lin, Y.F.; Aarts, M.G. The molecular mechanism of zinc and cadmium stress response in plants. Cell Mol. Life Sci. 2012, 69, 3187-3206. [CrossRef] [PubMed]

34. Tiwari, M.; Gupta, A.; Sharma, A.; Prasad, S.; Pandey, A.N.; Yadav, P.K.; Pandey, A.K.; Shrivastav, T.G.; Chaube, S.K. Role of Mitogen Activated Protein Kinase and Maturation Promoting Factor During the Achievement of Meiotic Competency in Mammalian Oocytes. J. Cell Biochem. 2018, 119, 123-129. [CrossRef] [PubMed]

35. Han, X.; Li, S.; Zhang, M.; Yang, L.; Liu, Y.; Xu, J.; Zhang, S. Regulation of GDSL Lipase Gene Expression by the MPK3/MPK6 Cascade and Its Downstream WRKY Transcription Factors in Arabidopsis Immunity. Mol. Plant-Microbe Interact. MPMI 2019, 32, 673-684. [CrossRef]

36. Shen, H.; Liu, C.; Zhang, Y.; Meng, X.; Zhou, X.; Chu, C.; Wang, X. OsWRKY30 is activated by MAP kinases to confer drought tolerance in rice. Plant Mol. Biol. 2012, 80, 241-253. [CrossRef] [PubMed]

37. Liu, J.; Chen, X.; Liang, X.; Zhou, X.; Yang, F.; Liu, J.; He, S.Y.; Guo, Z. Alternative Splicing of Rice WRKY62 and WRKY76 Transcription Factor Genes in Pathogen Defense. Plant Physiol. 2016, 171, 1427-1442. [CrossRef]

38. Sheen, J. Signal transduction in maize and Arabidopsis mesophyll protoplasts. Plant Physiol. 2001, 127, 1466-1475. [CrossRef]

39. Zhai, Z.; Sooksa-nguan, T.; Vatamaniuk, O.K. Establishing RNA interference as a reverse-genetic approach for gene functional analysis in protoplasts. Plant Physiol. 2009, 149, 642-652. [CrossRef] [PubMed]

40. Jiang, Y.; Deyholos, M.K. Functional characterization of Arabidopsis NaCl-inducible WRKY25 and WRKY33 transcription factors in abiotic stresses. Plant Mol. Biol. 2009, 69, 91-105. [CrossRef] [PubMed]

41. Kim, C.Y.; Zhang, S. Activation of a mitogen-activated protein kinase cascade induces WRKY family of transcription factors and defense genes in tobacco. Plant J. 2004, 38, 142-151. [CrossRef]

42. Chi, Y.; Yang, Y.; Zhou, Y.; Zhou, J.; Fan, B.; Yu, J.Q.; Chen, Z. Protein-protein interactions in the regulation of WRKY transcription factors. Mol. Plant 2013, 6, 287-300. [CrossRef] [PubMed]

43. Mao, G.; Meng, X.; Liu, Y.; Zheng, Z.; Chen, Z.; Zhang, S. Phosphorylation of a WRKY transcription factor by two pathogenresponsive MAPKs drives phytoalexin biosynthesis in Arabidopsis. Plant Cell 2011, 23, 1639-1653. [CrossRef] [PubMed]

44. Dong, J.; Chen, C.; Chen, Z. Expression profiles of the Arabidopsis WRKY gene superfamily during plant defense response. Plant Mol. Biol. 2003, 51, 21-37. [CrossRef] [PubMed]

45. Pecher, P.; Eschen-Lippold, L.; Herklotz, S.; Kuhle, K.; Naumann, K.; Bethke, G.; Uhrig, J.; Weyhe, M.; Scheel, D.; Lee, J. The Arabidopsis thaliana mitogen-activated protein kinases MPK3 and MPK6 target a subclass of 'VQ-motif'-containing proteins to regulate immune responses. New Phytol. 2014, 203, 592-606. [CrossRef]

46. Xiao, G.Z.; Wu, X.L.; Teng, K.; Chao, Y.H.; Li, W.T.; Han, L.B. [Hyperspectral Analysis and Electrolyte Leakage Inversion of Creeping Bentgrass under Salt Stress]. Guang Pu Xue Yu Guang Pu Fen Xi 2016, 36, 3630-3636. 
47. Hayat, S.; Hayat, Q.; Alyemeni, M.N.; Wani, A.S.; Pichtel, J.; Ahmad, A. Role of proline under changing environments: A review. Plant Signal Behav. 2012, 7, 1456-1466. [CrossRef] [PubMed]

48. Gaweł, S.; Wardas, M.; Niedworok, E.; Wardas, P. [Malondialdehyde (MDA) as a lipid peroxidation marker]. Wiadomosci Lekarskie (Warsaw Poland 1960) 2004, 57, 453-455.

49. Sparkes, I.A.; Runions, J.; Kearns, A.; Hawes, C. Rapid, transient expression of fluorescent fusion proteins in tobacco plants and generation of stably transformed plants. Nat. Protoc. 2006, 1, 2019-2025. [CrossRef] [PubMed]

50. Nelson, B.K.; Cai, X.; Nebenfuhr, A. A multicolored set of in vivo organelle markers for co-localization studies in Arabidopsis and other plants. Plant J. 2007, 51, 1126-1136. [CrossRef]

51. Zhu, Y.; Zuo, M.; Liang, Y.; Jiang, M.; Zhang, J.; Scheller, H.V.; Tan, M.; Zhang, A. MAP65-1a positively regulates H2O2 amplification and enhances brassinosteroid-induced antioxidant defence in maize. J. Exp. Bot. 2013, 64, 3787-3802. [CrossRef]

52. Liu, W.; Xiang, Y.; Zhang, X.; Han, G.; Sun, X.; Sheng, Y.; Yan, J.; Scheller, H.V.; Zhang, A. Over-Expression of a Maize NAcetylglutamate Kinase Gene (ZmNAGK) Improves Drought Tolerance in Tobacco. Front. Plant Sci. 2018, 9, 1902. [CrossRef] [PubMed]

53. Jiang, M.; Zhang, J. Water stress-induced abscisic acid accumulation triggers the increased generation of reactive oxygen species and up-regulates the activities of antioxidant enzymes in maize leaves. J. Exp. Bot. 2002, 53, 2401-2410. [CrossRef]

54. Ma, H.; Liu, C.; Li, Z.; Ran, Q.; Xie, G.; Wang, B.; Fang, S.; Chu, J.; Zhang, J. ZmbZIP4 Contributes to Stress Resistance in Maize by Regulating ABA Synthesis and Root Development. Plant Physiol. 2018, 178, 753-770. [CrossRef] [PubMed]

55. Xiang, Y.; Sun, X.; Bian, X.; Wei, T.; Han, T.; Yan, J.; Zhang, A. ZmNAC49 reduces stomatal density to improve drought tolerance in maize. J. Exp. Bot. 2020, 72, 1399-1410. [CrossRef] [PubMed]

56. Shi, B.; Ni, L.; Zhang, A.; Cao, J.; Zhang, H.; Qin, T.; Tan, M.; Zhang, J.; Jiang, M. OsDMI3 is a novel component of abscisic acid signaling in the induction of antioxidant defense in leaves of rice. Mol. Plant 2012, 5, 1359-1374. [CrossRef] [PubMed]

57. Jiang, M.; Zhang, J. Effect of abscisic acid on active oxygen species, antioxidative defence system and oxidative damage in leaves of maize seedlings. Plant Cell Physiol. 2001, 42, 1265-1273. [CrossRef]

58. Koca, H.; Bor, M.; Özdemir, F.; Türkan, I. The effect of salt stress on lipid peroxidation, antioxidative enzymes and proline content of sesame cultivars. Environ. Exp. Bot. 2007, 60, 344-351. [CrossRef]

59. Kumar, S.; Stecher, G.; Tamura, K. MEGA7: Molecular Evolutionary Genetics Analysis Version 7.0 for Bigger Datasets. Mol. Biol. Evol. 2016, 33, 1870-1874. [CrossRef] [PubMed] 